\title{
TESTAGEM INICIAL E CAPACIDADE DISCRIMINATÓRIA DA ESCALA “UFMG SYDENHAM'S CHOREA RATING SCALE (USCRS)"
}

\author{
Antônio Lúcio Teixeira-Jr1,2, Débora Palma Maia ${ }^{1}$, Francisco Cardoso ${ }^{1,2}$
}

\begin{abstract}
RESUMO - Recentemente desenvolvemos e validamos a escala "Universidade Federal de Minas Gerais (UFMG) Sydenham's Chorea Rating Scale" (USCRS) para avaliar sistematicamente os pacientes com coréia de Sydenham (CS). Neste estudo, examinamos 97 crianças e adultos com CS (média de idade \pm desvio padrão, 15,5 $\pm 5,9$; masculino/feminino, 31/66) acompanhados na Clínica de Distúrbios do Movimento da UFMG, empregando a escala USCRS. Os pacientes foram divididos em 4 grupos conforme a forma clínica apresentada: aguda $(n=19)$, recorrente $(n=17)$, persistente $(n=19)$ e remissão $(n=42)$. O escore médio \pm erro padrão na escala USCRS para cada um dos grupos foi, respectivamente, 47,7 $\pm 4,7$ para aguda, 29,5 $\pm 2,6$ para recorrente, 17,6 $\pm 3,1$ para persistente e 1,1 $\pm 0,2$ para remissão. Todas as comparações entre cada um dos grupos foram estatisticamente significativas $(p<0,05)$. Nossos resultados sugerem que a escala USCRS pode discriminar razoavelmente os grupos de pacientes com em diferentes formas clínicas de CS.
\end{abstract}

PALAVRAS-CHAVE: coréia de Sydenham, escala.

\section{The initial testing and the discrimination property of the UFMG Sydenham's Chorea Rating Scale (USCRS)}

\begin{abstract}
Recently we developed and validated the Universidade Federal de Minas Gerais (UFMG) Sydenham's chorea Rating Scale (USCRS) to systematically assess SC patients. In this study, we assessed 97 children and adults with SC (mean age \pm SD, $15.5 \pm 5.9$; male/female, 31/66) seen at the Movement Disorders Clinic at UFMG employing the USCRS. The patients were divided into 4 groups according to their clinical status: acute $(n=19)$, recu rrent $(n=17)$, persistent $(n=19)$ and remission $(n=42)$. The mean \pm SEM USCRS scores for each g roup were: $47.7 \pm 4.7$ for acute group, $29.5 \pm 2.6$ for recurrent group, $17.6 \pm 3.1$ for persistent group and 1.1 \pm 0.2 for remission group. All pair comparisons were statistically significant $(p<0.05)$. Our results indicate that the USRSC can reasonably discriminate groups of SC patients in different clinical stages of the disease.
\end{abstract}

KEY WORDS: Sydenham's chorea, rating scale.

A febre reumática é doença sistêmica inflamatória auto-imune que ocorre cerca de 2 a 4 semanas após a infecção das vias aéreas superiores pelo estreptococos $\beta$ hemolítico do grupo A, o Strepto coccus pyogenes'. A febre reumática acomete principalmente crianças na faixa etária escolar e constitui importante problema de saúde pública em países em desenvolvimento como o Brasil, onde a cardiopatia reumática, re presentada por valvopatias mitral e aórtica, é a principal causa de doença cardíaca em indivíduos com menos de 40 anos de idade ${ }^{2,3}$. A coréia de Sydenham (CS) é a manifestação ne u rológica da febre reumática, ocorrendo em cerca de $30 \%$ dos $\operatorname{casos}^{4-6}$. Caracteriza-se pela presença de movimentos involuntários arrítmicos, breves, que fluem de uma parte a outro do corpo de forma não estereotipada, daí o termo coréia, derivado do grego "choreus" que significa dançar. Os pacientes com CS exibem outros sinais neurológicos, como sacadas hipométricas, disartria, hipotonia, comprometimento da marcha e tiques ${ }^{4}$. Sintomas neuropsiquiátricos, como labilidade afetiva, hiperatividade e sintomas obsessivo-compulsivos, são também freqüentemente encontrados em pacientes com $\mathrm{CS}^{7,8}$. A observação sistemática da comorbidade de transto rnos neuropsiquiátricos, como o transtorno obsessivo-compulsivo e a síndrome de To u rette, na CS despertou interesse em diferentes grupos de pesquisa, que passaram a buscar evidências do papel da infecção estreptocóccica no desenvolvimento desses transtornos na infância7,8. Uma limitação dos estudos nessa área era a inexistência de

${ }^{1}$ Clínica de Distúrbios do Movimento do Hospital das Clínicas da Universidade Federal de Minas Gerais (UFMG) e ${ }^{2}$ Departamento de Clínica Médica da Faculdade de Medicina da UFMG, Belo Horizonte MG, Brasil.

Recebido 18 Fevereiro 2005, recebido na forma final 9 Maio 2005. Aceito 1 Junho 2005. 
escalas para a avaliação quantitativa da coréia. Recentemente desenvolvemos e validamos uma escala para a avaliação sistemática dos pacientes com CS denominada "Universidade Federal de Minas Gerais (UFMG)' Sydenham's Chorea Rating Scale (USCRS)" ${ }^{\prime}$. A escala USCRS mostrou ser um instrumento com elevadas confiabilidade inter-examina dor e consistência interna ${ }^{9}$.

Neste trabalho, apresentamos a versão em língua portuguesa da escala USCRS, publicada inicialmente em inglês ${ }^{9}$, e avaliamos a capacidade discriminatória da escala frente a diferentes grupos de pacientes com CS.

\section{MÉTODO}

Foram estudados 97 crianças e adultos (média de idade \pm desvio padrão, 15,5 \pm 5,9; masculino/feminino, 31/66) com CS acompanhados na Clínica de Distúrbios do Movimento da UFMG no período de janeiro de 2003 a novembro de 2004. O diagnóstico de CS foi conferido a pacientes que apresentaram coréia de início agudo, preencheram os critérios modificados para febre reumática aguda10 e outras causas de coréia foram cuidadosamente excluídas ${ }^{4,11}$.

Cada paciente foi entrevistado e examinado individualmente, recebendo uma pontuação conforme a escala USCRS (disponível no site da Academia Brasileira de Neurologia - www.abneuro.org). Brevemente, a escala USCRS foi desenvolvida para fornecer descrição quantitativa detalhada do funcionamento do paciente com CS nas atividades da vida diária, da presença de alterações comportamentais e do comprometimento da função motora. A escala compreende 27 itens, cada um pontuado de 0 (ausência de sinal ou sintoma) a 4 (sintoma grave ou de incapacidade funcional). Alguns itens da escala USCRS, como o movimento ocular de perseguição e a agilidade de membros inferiores, basearamse nas escalas "Unified Huntington's Disease Rating Scale (UHDRS)" 12 e "Unified Parkinson's Disease Rating Scale (UPDRS)"13. Outros itens da escala USCRS, como tônus muscular, foram formulados pelos autores a partir de experiência clínica própria.

Os pacientes com CS foram também classificados em um de quatro grupos possíveis conforme o estágio clínico da doença: aguda, recorrente, persistente, remissão. A CS aguda compreende os casos com coréia em atividade a despeito do tratamento anti-coréico, cuja duração não exceda dois anos. A CS em remissão envolve os pacientes em que houve resolução dos movimentos coréicos, mesmo sem uso de medicamento contra movimento anormal. A CS recorrente inclui os pacientes que, após período de remissão, apresentaram novo episódio de coréia. A CS persistente compreende os casos em que os movimentos involuntários são persistentes por mais de 2 anos apesar do tratamento anti-córeico ${ }^{11}$.

Para análise estatística realizou-se análise de variância
(ANOVA) e pós-teste de Tukey para múltiplas comparações, estabelecendo-se o nível de significância em $\mathrm{p}<0,05$.

\section{RESULTADOS}

Os pacientes foram divididos em 4 grupos conforme o estágio clínico da doença: aguda $(n=19)$, recorrente $(n=17)$, persistente $(n=19)$ e remissão $(n=42)$. Os escores médios \pm erro padrão na escala USCRS para cada um dos grupos foram: 47,7 $\pm 4,7$ para o grupo na forma aguda, 29,5 $\pm 2,6$ para a forma recorrente, $17,6 \pm 3,1$ para a forma persistente e 1,1 \pm 0,2 para a forma remissão (Figura). Todas as comparações entre pares de grupos foram estatisticamente significativas $(p<0,05)$.

\section{DISCUSSÃO}

A patogênese da CS parece estar relacionada à disfunção dos núcleos da base mediada por processos auto-imunes, principalmente auto-anticorpos, após a infecção estreptocóccica ${ }^{14,15}$. Uma série de estudos de neuroimagem estrutural e funcional confirmou o comprometimento dos núcleos da base na $\mathrm{CS}^{16}$. A análise dos sintomas neurológicos e comportamentais exibidos por pacientes reforça a concepção de comprometimento dos núcleos da base e, por conseguinte, dos diferentes circuitos frontoestriatais na $\mathrm{CS}^{17,18}$. A presença de movimentos involuntários como coréia e, ocasionalmente, tiques indica o comprometimento do circuito fronto-estriatal motor. A ocorrência de sacadas hipométricas sugere interferência no circuito óculo-motor. Como os sintomas obsessivo-compulsivos e de hiperatividade são comuns na CS, há possivelmente comprometimento de circuitos fronto-estriatais não-motores, como os circuitos órbito-frontal e dorso-lateral ${ }^{18}$. Postula-se, então, que a variabilidade dos quadros

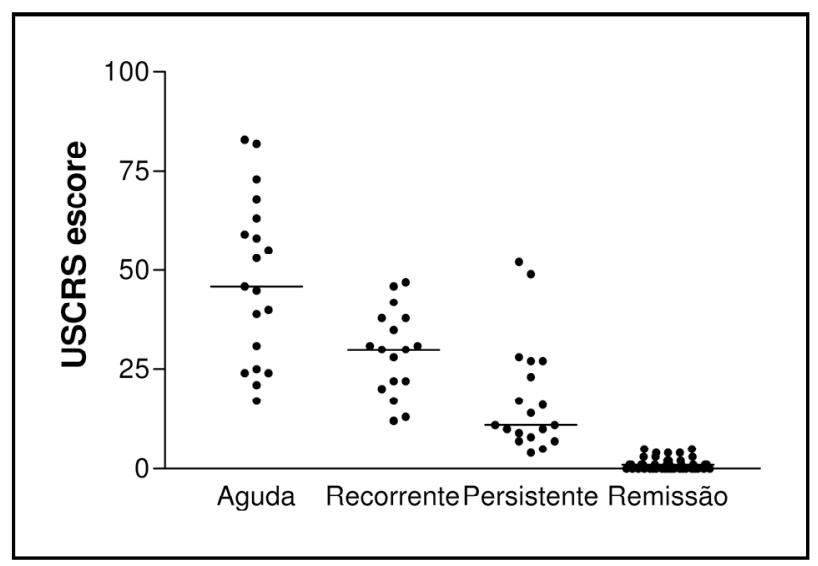

Figura. Pontuação na escala USCRS ("UFMG Sydenham's Chorea Rating Scale") apresentada por pacientes com diferentes formas clínicas de coréia de Sydenham. 
clínicos apresentados pelos pacientes com CS poderia refletir disfunção seletiva dos diferentes circuitos fronto-estriatas mediada pelos processos autoimunes pós-estreptocóccicos ${ }^{19}$.

A escala USCRS foi elaborada justamente no sentido de avaliar as diferentes dimensões sintomáticas da CS. Em estudo anterior, mostrou-se um instrumento confiável, exibindo valores elevados, entre 0,86 e 1,0, de correlação intra-classe para todos os itens da escala, e de alta consistência interna com $\alpha$-Cronbach igual a 0,979. Na análise de composição fatorial da escala, dois fatores emergiram, um contendo os itens de alterações comportamentais e outro com os demais itens. Isso poderia indicar comp rometimento diferencial dos circuitos fronto-estriatais na $\mathrm{CS}^{9}$.

Os resultados do presente estudo sugerem que a escala USRSC pode discriminar razoavelmente grupos de pacientes com CS em diferentes estágios clínicos da doença. Salienta-se, entretanto, que há superposição entre alguns valores individuais apresentados por pacientes nos grupos com CS aguda, CS recorrente e CS persistente (Figura).

Os pacientes com CS aguda evidenciaram os sintomas mais graves conforme a pontuação na escala USCRS. Os pacientes com CS recorrente apresentaram sintomas menos intensos que os pacientes com CS aguda, mas mais significativos que os com CS persistente. Considerando-se a hipótese sobre a patogênese da CS que postula a disfunção dos núcleos da base mediada por auto-anticorpos ${ }^{14,15}$, esses dados são interessantes pois sugerem que o envolvimento dos núcleos da base é mais intenso justamente nas fases em que os processo auto-imunes estão mais ativos. Em relação à CS persistente, embora alguns pacientes também apresentem auto-anticorpos ${ }^{14}$, é possível que a cronificação dos sintomas se relacione com outra modalidade de disfunção imunológica, lesões estruturais ou bioquímicas ocorridas na fase aguda da CS.
Concluindo, a escala USCRS é um instrumento promissor para a quantificação de sinais e sintomas clínicos da CS, assim como para a avaliação do impacto funcional sobre crianças e adultos.

\section{REFERÊNCIAS}

1. Stollerman GH. Rheumatic fever in the 21st century. Clin Infect Dis 2001;33:806-814.

2. Mendez GF, Cowie MR. The epidemiological features of heart failure in developing countries: a review of the literature. Int J Cardiol 1999; 80:213-219.

3. Silva $\mathrm{CH}$ and the Pediatric Committee of the São Paulo Pediatric Rheumatology Society. Rheumatic fever: a multicenter study in the state of São Paulo. Rev Hosp Clin Fac Med S Paulo 1999;54:85-90.

4. Cardoso F, Eduardo C, Silva AP, Mota CC. Chorea in fifty consecutive patients with rheumatic fever. Mov Disord 1997;12:701-703.

5. Goldenberg J, Ferraz MB, Fonseca AS, Hilario MO, Bastos W, Sachetti S. Sydenham chorea: clinical and laboratory findings: analysis of 187 cases. Rev Paul Med 1992;110:152-157.

6. Araújo AP, Pádua PA, Maia-Filho HS. Management of rheumatic chorea: an observational study. Arq Neuropsiquiatr 2002;60:231-233.

7. Swedo SE. Sydenham's chorea. A model for childhood autoimmune neuropsychiatric disorders. JAMA 1994;272:1788-1791.

8. Mercadante MT, Busatto GF, Lombroso PJ, et al. Psychiatric symptoms of rheumatic fever. Am J Psychiatry 2000; 157:2036-2038.

9. Teixeira-Jr AL, Maia DP, Cardoso F. The UFMG Sydenham's Chorea Rating Scale (USCRS): reliability and consistency. Mov Disord 2005; 20:585-591.

10. Special Writing Group of the Committee on Rheumatic Fever, Endocarditis, and Kawasaki Disease of the Council on Cardiovascular Disease in the Young of the American Heart Association. Guidelines for the diagnosis of rheumatic fever: Jones criteria, 1992 update. JAMA 1992; 268:2069-2073.

11. Cardoso F, Vargas AP, Oliveira LD, Guerra AA, Amaral SV. Persistent Sydenham's chorea. Mov Disord 1999;14:805-807.

12. Huntington Study Group. Unified Huntington's Disease Rating Scale: reliability and consistency. Mov Disord 1996;11:136-142.

13. Fahn S, Elton RL, and members of the UPDRS Development Committee. Unified Parkinson's Disease Rating Scale. In Fahn S, Marsden CD, Goldstein M, (eds). Recent developments in Parkinson's disease, II. Florham Park: MacMillan Healthcare Information, 1987;293-304.

14. Church AJ, Cardoso F, Dale RC, Lees AJ, Thompson EJ, Giovannoni G. Anti-basal ganglia antibodies in acute and persistent Sydenham's chorea. Neurology 2002;59:227-231.

15. Kirvan CA, Swedo SE, Heuser JS, Cunningham MW. Mimicry and autoantibody-mediated neuronal cell signaling in Sydenham chorea. Nat Med 2003; 9: 914-920.

16. Dale RC. El estreptococo piógenes y el cerebro: viviendo con el enemigo. Rev Neurol 2003;37:92-97.

17. Tekin S, Cummings JL. Frontal-subcortical neuronal circuits and clinical neuropsychiatry: an update. J Psychosom Res 2002;53:647-654.

18. Teixeira-Jr AL, Cardoso F. Neuropsiquiatria dos núcleos da base: uma revisão. J Bras Psiquiatr 2004;53:153-158.

19. Mercadante MT, Hounie AG, Diniz JB, Miguel EC, Lombroso PJ. The basal ganglia and immune-based neuropsychiatric disorders. Psychiatr Ann 2001;31:534-540.21. 